\title{
Studying the intersystem crossing rate and triplet quantum yield of meso-substituted porphyrins by means of pulse train fluorescence technique
}

\author{
Tiago Gualberto Bezera de Souza ${ }^{a}$, Marcelo Gonçalves Vivas ${ }^{b}$, Cleber Renato \\ Mendonça $^{a}$, Shane Plunkett ${ }^{c}$, Mikhail A. Filatov ${ }^{c}$, Mathias O. Senge ${ }^{*}$ and Leonardo \\ De Boni ${ }^{* a}$
}

a Instituto de Física de São Carlos, Universidade de São Paulo, CP 369, 13560-970 São Carlos-SP, Brazil

b Instituto de Ciência de Tecnologia, Universidade Federal de Alfenas, Cidade Universitária-BR 267 Km 533, $37715-$ 400 Poços de Caldas, MG, Brazil

c School of Chemistry, SFI Tetrapyrrole Laboratory, Trinity Biomedical Sciences Institute, 152-160 Pearse Street, Trinity College Dublin, Dublin 2, Ireland

Dedicated to Professor Kevin M. Smith

Received date (to be automatically inserted after your manuscript is submitted)

Accepted date (to be automatically inserted after your manuscript is accepted)

\begin{abstract}
Excited state dynamics, particularly intersystem crossing, of a set of meso-substituted porphyrins bearing different electron-donor and acceptor groups was studied by pulse train fluorescence technique. Triplet quantum yield was found to be critically dependent on the nature of meso-substituents in the porphyrin system. Porphyrins with meso methoxyphenyl groups were found to show high triplet quantum yields $\left(\phi_{T}\right.$ between 0.70 and 0.81 ). Moreover, the quantity of methoxyphenyl groups and the substitution pattern directly influence $\phi_{T}$. Alternatively, porphyrins attached to nitrophenyl group possess low triplet quantum yield values $(\sim 0.3)$. The observed structure-properties relationships suggest new ways for tuning the optical properties of porphyrins via chemical modification.
\end{abstract}

KEYWORDS: relaxation times / quantum yields / triplet state/ donor/acceptor groups / excited state spectroscopy / porphyrins 


\section{INTRODUCTION}

The remarkable optical properties of porphyrins continue to attract significant attention, mainly due to their large excited state absorption [1,2] (particularly in the near-IR part of the spectrum [3]), two-photon absorption [4,5], fast intersystem crossing (ISC) [6], excellent optical stability and biocompatibility [7]. As a result of these features porphyrins have extensively been studied with respect to the development of novel optoelectronic and photonic devices [8]. One of the most promising characteristics of porphyrins optical properties that allow their application in technological devices is the formation of long-lived triplet excited states upon photoexcitation. Such a transfer of excited state populations between singlet and triplet states is also known as intersystem crossing (ISC).

Efficient ISC is important for distinct photonic processes, e.g., optical power limiting (OPL) which attracts attention due to growing development of new laser systems with large pulse energies [9]. Very fast excited state absorption and high singlet to triplet conversion rates [10-12] make porphyrins a unique class of compounds for this application [1315]. Besides OPL, the ISC features of porphyrins are also important for other applications such as photodynamic therapy (PDT) [1,16], dye sensitized solar cells [17] and photon up-conversion devices [18].

The ISC rate $\left(k_{i s c}\right)$ is one of the transition parameters that control the population dynamics between singlet and triplet excited states. Specifically, it is the reciprocal value of $k_{i s c}$ gives the characteristic time to electron population decay from the first singlet excited state to the first triple excited $\left(\tau_{i s c}=1 / k_{i s c}\right)$ [19]. Usually, excited state dynamics of porphyrins can be illustrated by a three level Jablonski energy diagram.

It is well known that the optical properties of porphyrins are affected by the nature of the central metal and meso substituents and thus, adjusting the substitution pattern, it can be used as a tool for privilege a specific type of excited states relaxation process. For example, Gonçalves et al. [20] demonstrated that the insertion of zinc in sulfonated porphyrin free-bases drastically reduced the ISC rate from $13 \mathrm{~ns}$ [21] to $2.2 \mathrm{~ns}$ [20]. Sampaio et al. [22] have shown that free-base 5,10,15,20-tetra(4-pyridyl)porphyrin $\left(\mathrm{H}_{2} \mathrm{TP} \mathrm{y}\right)$ with ruthenium(II) ions bound at the peripheral groups of the macrocycle increases the ISC time about twofolds compared to porphyrins without Ru(II) (from $14 \mathrm{~ns}$ to $25 \mathrm{~ns}$ ). This behavior is opposite to that of porphyrins with $\mathrm{Ru}(\mathrm{II})$ inserted in macrocycle core. Sulfonated free-base porphyrins possessing halogenated substituents [23] exhibited increased intersystem crossing quantum yields ( $\left.\phi_{i s c}\right)$ [21]. For example, $\phi_{T}$ increased from 0.77 to 0.84 upon introduction of two fluorine atoms and to 0.85 and 0.91 for one and two chlorine atoms, respectively. Moreover, halogenation of the porphyrin does not only affect the ISC rate but also fluorescence parameters, making these compounds suitable for theranostics applications.

In this context, the present work is focused on gaining a better understanding of the relationship between mesosubstitution pattern and ISC rates of porphyrins. To achieve this, a set of nineteen porphyrins with different donor and acceptor groups were studied by pulse train fluorescence technique developed recently in our group [24].

\section{EXPERIMENTAL}

\section{Samples}

A set of nineteen porphyrins with different meso-substitution patterns have been prepared according to published procedures $[25-33,35]$ and were used in this study (Figure 1).

$<$ Figure 1>

Different meso substituents affect the electronic density distribution in the porphyrin macrocycle, thus modifying its excited state properties. The set 1-19 of porphyrins varying in their meso-substitution pattern was therefore prepared and investigated in order to reveal intersystem crossing rates along with other spectroscopic parameters. The aim was to 
provide guidelines for choosing promising candidates for applications which involve porphyrin triplet excited state formation, i.e. in photodynamic therapy [34]. For spectroscopic studies, the corresponding porphyrins were dissolved in dimethyl sulfoxide (DMSO) which was chosen for its low volatility and high solubility of the porphyrins studied therein.

\section{Experimental set-up}

The excitation system employed to measure intersystem crossing rate of all porphyrins studied in this work includes a frequency-doubled $(532 \mathrm{~nm})$, mode-locked and Q-Switched Nd:YAG laser. This system delivers a sequence of about 30 mode-locked pulses modulated by the Q-switched gain envelope (pulse train). Temporal width of each individual pulse is about 100 ps FWHM, limited by the intracavity active mode-locked of the laser system. The pulses are separated from each other by approximately $13.2 \mathrm{~ns}$. At the laser output, a characteristic profile observed is shown in Figure 2a. The Q-Switch module device is able to tune the pulse repetition rate from 3 up to $800 \mathrm{~Hz}$. For the experiments described herein, the repetition rate was set at $100 \mathrm{~Hz}$, that delivered an output power of about $30 \mathrm{~mW}$ for the whole pulse train.

$<$ Figure 2>

Two distinct laser pulse configurations of the laser system were used for sample excitation in fluorescence measurements (Fig. 2b). For lifetime measurements, a single pulse was extracted from the pulse train with the aid of a Pockels cell (PC), located between two crossed polarizers, delivering up to $2 \mathrm{~mW}$ at $100 \mathrm{~Hz}$. For ISC rate measurements, the PC was turned off and the whole pulse train was used. In both configurations, the laser beam pass through a spatial filter in order to obtain a Gaussian spatial profile, expanded by a telescope, and then focused by a lens with a focal distance of $12 \mathrm{~cm}$. The position of the sample (z) was adjusted with respect to the focal point to avoid a fluorescence saturation effect. Thus, the samples were always separated from the focal position by a well-known $\mathrm{z}$ distance, which allows to calculate a defined Gaussian beam waist $(w(z))$ with respect to the one in focal position $\left(w_{0}\right)$, required to determine the light intensity. In both cases, the sample's fluorescence was collected perpendicularly to the excitation beam through a $1 \mathrm{~mm}$ diameter optical fiber, positioned close to the excitation spot an optical filter was used to completely cut off the excitation light $(532 \mathrm{~nm}$ ). The optical fiber was directing the fluorescence signal to a $\sim 700 \mathrm{ps}$ rise time silicon photodetector (Fig. 2b, D). The photodetector electrical signal was acquired by a $1 \mathrm{GHz}$ oscilloscope and recorded by a computer. The procedure of measuring the sample fluorescence induced by pulse train is referred as pulse train fluorescence technique (PTF) [22], while the one using a single pulse is referred as time-resolved fluorescence technique (TRF).

The TRF technique indirectly probes porphyrin triplet excited states by monitoring the cumulative depletion of fluorescence signal, as a function of the laser pulse number, which is dependent on the population of the first excited state. The fluorescence induced by the pulse decreases due to the increase of the population in the triplet state over time. However, with the pulse train periodically building-up population in the first singlet excited state, in a time close to the one needed for a complete depletion of that state, the portion of excited state population transferred into triplet state, and consequently its effects on the depletion on porphyrin fluorescence, becomes significant.

Besides PTF and TRF measurements, additional experiments were performed to measure the absorption and emission spectra of the compounds studied. Absorption spectra were measured using a SHIMADZU UV-1800 spectrophotometer with $1 \mathrm{~nm}$ accuracy using a $2 \mathrm{~mm}$ quartz cuvette. Fluorescence spectra were recorded on a HITACHI F7000 fluorimeter.

\section{Rate equation model}


In order to determine the intersystem crossing time by monitoring the fluorescence signal pattern induced by the pulse train laser configuration for a set of porphyrin molecules, a three energy level model was considered, as illustrated by a Jablonski diagram (Fig. 3). Thus, knowing that the fluorescence signal intensity is proportional only to the population of the first singlet excited state $S_{l}$, it is essential to determine the population of this state as a function of the pulse fluency (pulse train envelope) and time (relaxation processes). Determination of the population dynamics between states requires some other photophysical parameters to be measured. Such parameters, which are essential to solve the population dynamics using the rate equation model, are the absorption cross-section, $\sigma_{01}$, of the ground state $\left(S_{0}\right)$ to the first excited $\left(S_{l}\right)$ and the fluorescence lifetime $\left(\tau_{f}\right)$, respectively. They can be obtained using other techniques such as linear absorption and time-resolved fluorescence measurements. Measurement of these parameters for each sample allows for the determination of the intersystem crossing rate, $\tau_{i s c}$, as the only adjustable parameter one the rate equation model.

$<$ Figure $3>$

Certain considerations were made in order to simplify the rate equations describing the population dynamics. First, higher excited state transitions can be neglected from the model because their relaxation happens on ps timescale, i.e. these states are totally depopulated within a laser pulse (100 ps) of the pulse train. Second, the emissive relaxation process from the triplet state, $T_{l}$, to $S_{0}$, i.e. a phosphorescent process, was ignored in this model. The timescale of this process $\left(\tau_{\text {phos }}=1 / k_{\text {phos }}\right)$, which depends on the decay rates combination $\left(k_{\text {rad }}^{T}\right.$ and $\left.k_{\text {nrad }}{ }^{T}\right)$ from $T_{1}$ to $S_{0}$, is much longer than that of the fluorescence; normally it is on the timescale of microseconds. In other words, during the pulse train interaction with the porphyrin molecule, there is not enough time for repopulation of the ground state, subsequently trapping the electrons in the $T_{l}$ state, and consequently decreasing the effective fluorescence signal during the pulse train window.

Consequently, the transition of the electron population dynamics in each state $(n)$ between the energy states is simulated through the simplified rate equations. Additionally, the numerical solution of the rate equations require a boundary condition provided by a population normalization $\left(\sum_{i} n_{i}(t)=1\right)$ and stating an initial condition $\left(\mathrm{n}_{\mathrm{s} 0}(0)=1\right)$. Thus, the rate equations corresponding to the system depicted in Fig. 3 can be simplified as follows (equation 1):

\section{Equation 1}

$\frac{d n_{S_{0}}(t)}{d t}=-W_{01}^{s} n_{S_{0}}(t)+\left(k_{f}-k_{i s c}\right) n_{S_{1}}(t) ;$

$\frac{d n_{S_{1}}(t)}{d t}=+W_{01}^{s} n_{S_{0}}(t)-k_{f} n_{S_{1}}(t)$

$\frac{d n_{T_{1}}(t)}{d t}=+k_{i s c} n_{S_{1}}(t)$

Following the rate equations, one can notice that the variation of the population as a function of time depends on the initial transition probability between the ground and first singlet excited state $\left(W_{01}=\phi \sigma_{01}\right)$, related with the transition absorption cross-section $\sigma_{01}(\lambda)$ and the excitation light fluency $(\phi=I / h v)$. The latter is defined by each pulse of the pulse train envelope. Thus, when the first pulse of train arrives on the sample, population from $S_{0}$ is transferred to $S_{l}$ proportional to the intensity of that pulse, the absorption cross-section, and to the initial population of the ground state $\left(n_{S O}(0)=1\right)$. Subsequently, a fraction of the population transferred to $S_{I}$ may relax back to the ground state with a rate defined by the difference between the fluorescence and intersystem crossing mechanisms. At the same time, a fraction of the population in $\mathrm{S}_{1}$ undergoes intersystem crossing and populates the triplet state. Once that population fraction is 
transferred to the $T_{1}$ state, it becomes trapped in this state for a period of the phosphorescence lifetime. The second pulse arrives to the sample after $13.2 \mathrm{~ns}$ and excites another population fraction. For this pulse, $S_{0}$ population will be defined as $n_{S 0}(13.2 \mathrm{~ns})<1$, since a certain fraction of the excited states population is trapped in $T_{1}$. Consequently, the population transferred to $S_{l}$ will depend on the intensity of the second pulse, absorption cross-section and on a current population fraction of the ground state. This process will occur until the last pulse of the envelope excites the sample. Thus, solving numerically the set of rate equations (equation 1) using the pulse train envelope as fluency input, allows to simulate the population as a function of the time in all states including the first excited state, which is the one responsible for the fluorescence signal. Determination of $\tau_{i s c}$ (intersystem crossing rate constant) was achieved by considering it as the only free parameter in Eq. 1 to adjust the experimental curve. It determines the population fraction transferred from $S_{1}$ to $T_{1}$ and stays trapped on the triplet state, which, consequently, will not contribute to the fluorescence signal anymore on the pulse train timescale. It is important to note that with the absorption cross-section ( $\sigma_{01}$ of the $S_{0}$ to the $S_{l}$ ) and the fluorescence lifetime $\left(\tau_{f}\right)$ measured, the intersystem crossing rate constant is the only parameter to be elucidated from the rate equations.

This effect is illustrated in Fig. 4a. It shows the fluorescence signal (solid black line) and the pulse train reference signal (grey dashed line). A comparison of the fluorescence signal envelope with the reference shows that the fluorescence signal does not follow the reference signal as the time interaction increases (function of the time). At the beginning $(\mathrm{t}<100 \mathrm{~ns})$, the fluorescence signal was proportional to the intensity of the pulse reference. This occurred because the number of molecules transferred to the triplet state was still small due to the low intensity of the laser pulses and it did not affect the fluorescence process considerably. However, as more and more pulses interacted with the sample, the fluorescence process is no longer proportional to the intensity of the corresponding pulse reference $(\mathrm{t}>$ $100 \mathrm{~ns}$ ). Thus, the depletion of the fluorescence signal takes place due to a population transfer from $S_{1}$ to $T_{1}$ via ISC, which, consequently, increases $T_{1}$ population [24].

Another way to describe the depletion of fluorescence with respect to the pulse train envelope (pulse index) is by the ratio between the fluorescence signal (solid black line) and the pulse train reference signal (grey dashed line), as

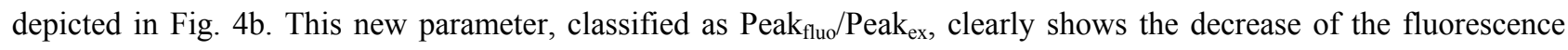
signal as a function of the pulse index (time). In the beginning, the Peak $\mathrm{fluo}_{\mathrm{fl}} / \mathrm{Peak}_{\mathrm{ex}}$ was close to 1 and represented a small fraction of the excited state population transferred to $T_{l}$. As the pulse index increased, the Peak $\mathrm{fluo}_{\mathrm{flu}} / \mathrm{Peak}_{\mathrm{ex}}$ increases, representing depletion of $S_{l}$ population. The $\mathrm{Peak}_{\mathrm{fluo}} / \mathrm{Peak}_{\mathrm{ex}}$ carries indirect information about the triplet state population. It is the input parameter to be modeled by the rate equations and will be considered further. The dependency of this ratio as a function of the pulse number will be adjusted by the rate equation model and gives, as only adjustable parameter, the intersystem crossing time $\left(\tau_{i s c}\right)$.

$<$ Figure $4>$

\section{RESULTS AND DISCUSSION}

The ground state cross-section, which is an important parameter to describe the excited state dynamic, was obtained from the linear relation with the absorption spectrum. Figure 5 shows the ground state absorption cross-section for porphyrin 4. Additionally, the fluorescence spectrum for the $S_{l}$ is shown (dash-dot curve). All the other samples possess similar absorption and fluorescence spectra which can be found in the Supporting Information (Fig. SI 1).

$<$ Figure $5>$

As is known, porphyrin choromophores show a characteristic absorption spectrum, consisting of transition with high oscillation strength known as the B-band at around $420 \mathrm{~nm}$ and, usually, four consecutive bands with lower intensity 
known as the Q-band (between 500 and $650 \mathrm{~nm}$ ). Metalloporphyrin (18 and 19) spectra exhibit only two Q-bands. Different meso-substituents change the position of the bands slightly, but may change significantly the oscillation strength of B- and Q-bands. As mentioned, $\sigma_{01}$ an input parameter in Eq. 1, was obtained from the values of absorption at $532 \mathrm{~nm}$.

The fluorescence quantum yield $\left(\phi_{f}\right)$, or radiative quantum yield $\left(\left(\phi_{r}\right)\right.$, of the samples was measured in solution at low concentration (to avoid re-absorption) using the method described by Brouwer [36]. Hematoporphyrin was used as a reference sample $\left(\phi_{f}^{\text {ref }}=0.08\right)$ [37]. The fluorescence spectrum of the reference and samples were measured by exciting both at the same wavelength within the Q-band region. The fluorescence quantum yields of the samples were obtained from Equation 2:

Equation 2

$$
\Phi_{f}=\Phi_{f}^{r \odot f} \times \frac{\int_{\lambda_{0}}^{\lambda_{f}} F(\lambda) d \lambda}{\int_{\lambda_{0}}^{\lambda_{f}} F_{r e f}(\lambda) d \lambda} \times \frac{f_{r \in f}}{f} \times \frac{n^{2}}{n_{r \oplus f}^{2}}
$$

in which, $\mathrm{F}(\lambda)$ is the fluorescence spectrum, $\mathrm{f}=1-10_{E x}^{-\mathrm{A}(\lambda)}$ is the absorption factor, $\mathrm{A}\left(\lambda_{E x}\right)$ is the absorbance in the excitation wavelength and $n$ is the refraction index of solvent. The calculated fluorescence quantum yield values for all molecules investigated are given in Table 1. The values observed for all porphyrins dissolved in DMSO are less than 0.08 , with the highest for porphyrin 5 (0.074).

Time-resolved fluorescence data provided another input parameter to Eq. 1, the fluorescence decay rate:

\section{Equation 3}

$$
k_{f}=k_{r}+k_{u r}+k_{t s e}
$$

which describes all the possible paths (decay rates) to deplete $S_{l}$; i.e. radiative $k_{r}=\phi_{f} / \tau_{f}$, non-radiative $k_{n r}-k_{f}-k_{r}-k_{i s c}$ (or $k_{n r}=1 / \tau_{n r}$, in which, $\tau_{n r}$ is the non-radiative lifetime), and intersystem crossing $k_{i s c}=1 / \tau_{i s c}$. Although it is more common to express $k_{f}$ by its reciprocal value, the fluorescence lifetime $\left(\tau_{f}=1 / k_{f}\right)$. The TRF technique measures and records the temporal evolution of the sample fluorescence induced by a single pulse excitation, as described before. Figure 6 illustrates the fluorescence decay $\left(\tau_{f}\right)$ obtained from the TRF technique for selected samples. Once the fluorescence lifetime and the fluorescence quantum yield are obtained, the radiative lifetime $\left(\tau_{r}=1 / k_{r}\right)$ can be determined directly. The single pulse in the TFR technique is set at a repetition rate of $100 \mathrm{~Hz}$, and each recorded curve is a mean value obtained in one minute of acquisition. The mono-exponential function was used to fit the TRF data, and $\tau_{f}$ is obtained for all samples (Table 1). For convenience, only part of the data is shown (Fig. 6). Complete data can be found in the Supporting Information (Fig. SI2).

$<$ Figure 6>

The fluorescence lifetime for most of the samples was found to be between 10-14 ns. However, the molecules 3 (7.7 $\mathrm{ns}), \mathbf{2}(4.6 \mathrm{~ns}), \mathbf{1 8}(3.1 \mathrm{~ns})$, and $\mathbf{1 9}(2.9 \mathrm{~ns})$ presented a significant reduction of fluorescence lifetimes. Thus, the nature of the meso-substituents has different effects on the excited state lifetimes depending on their nature and strength. Moreover, the pattern of substitution in the meso-aryl groups (para or ortho) also affects the excited state dynamics $[38,39]$.

Regarding porphyrins 18 and 19, the drastic decrease in the fluorescence lifetime as well as a reduction of fluorescence quantum yield is associated with the presence of a metal ion in the porphyrin core. It has previously been shown that central metals enhance the non-radiative channels [40]. On the other hand, for molecules 3 and 2 , the reduction of the fluorescence lifetime can be explained by the electron-withdrawing effect of the nitrophenyl group. These groups promote higher extension of $\pi$-conjugation in the excited state compared to the ground state due to their 
strong electron-acceptor character, resulting in an increase of radiative decay rates [41]. At the same time, in the case of 2 compared to 3, a stronger decrease in the fluorescence lifetime was observed. This is due to the fact that the nitrophenyl group in $\mathbf{2}$ is present in only one meso-position, while compound $\mathbf{3}$ contains two such residues.

$<$ Table 1>

This charge asymmetry in $\mathbf{2}$ causes weaker electron delocalization in the porphyrin, enhances the non-radiative decay rate and decreases the radiative decay rate, as reported in Table 1. On the other hand, the charge distribution in 2 becomes more localized on the porphyrin ring than in $\mathbf{3}$ (in which two nitrophenyl groups are symmetrically attached to the macrocycle) contributing to the reduction of the charge separation. These results can also be explained in light of Fermi's Golden Rule, in which an increase in the transition dipole moment (directly related with an increase of charge separation) contributes to a higher radiative decay rate, as observed when we compared the radiative decay rates for the porphyrins 3 and 2. A similar effect was also observed for porphyrins $\mathbf{1 8}$ and 19, since the incorporation of metal ions causes an increase of charge density on the porphyrin ring [42], which produces an increase in non-radiative decay rates concomitantly with a decrease of the fluorescence quantum yield.

In order to measure the intersystem crossing rate by monitoring the fluorescence of the sample, the pulse train fluorescence technique was configured at a repetition rate of $100 \mathrm{~Hz}$, and each measurement was performed at 1 minute acquisition time. Figure 7 displays the $\mathrm{Peak}_{\text {fluo }} / \mathrm{Peak}_{\mathrm{ex}}$ ratio as a function of the pulse index of the pulse train for compound 19. Curves similar to this one are presented in the Supporting Information for the other samples (Fig. SI3). This curve composes an average of ten measurements indicating that the major dispersion in the measurements occurs at the beginning of the pulse train, for pulses with lower intensity, which produces a small fluorescence signal (see error bars). For each individual pulse of the "train", below saturation regime of intensity $\left(I_{s a t}=h \nu / \sigma(\lambda) \tau_{f}\right)$, the fluorescence signal must be proportional to the pulse strength. However, after several pulses interact with the sample, the ratio is no longer constant. This effect, i.e. the decrease of the fluorescence signal, can be clearly observed by plotting the ratio between the intensities of the fluorescence peak and excitation pulse peak as a function of the number of interacting pulses.

$<$ Figure $7>$

Having $\sigma_{01}$ at $532 \mathrm{~nm}$ and $\tau_{f}$ evaluated for all samples, the intersystem crossing rate can be obtained from the rate equations (Equation 1). The rate equations were numerically solved to simulate the FTP data and obtain the value of $\tau_{\text {ts }}$, which best fits (solid line) the experimental data shown in Figure 7. The results are summarized in Table 1. As well as the transition parameter measured with the experiments described, other parameters can also be easily computed from them, for instance the fluorescence quantum yield, the radiative decay rate, the non-radioactive decay rate from equation 3 , and the intersystem crossing efficiency $\phi_{T}=\phi_{f}\left(k_{t s c} / k_{r}\right)$.

Regarding the obtained triplet quantum yields, compounds $15(0.70), 16(0.75)$ and $17(0.81)$ exhibit particularly higher values. This makes them good candidates for applications in OPL and photodynamic therapy. It is observed that the increase of $\phi_{T}$ is directly related to the number of methoxyphenyl groups connected to the porphyrin macrocycle. The methoxyphenyl group is a strong electron donating group, causing a reduction in the $\pi$-electron delocalization which contributes to the decrease of the radiative and non-radiative decay rates and the increase of the intersystem crossing rate (Table 1). As seen from Table 1, porphyrins with smaller $\phi_{T}$ values contain nitrophenyl groups attached to the meso-positions. These three porphyrins $(2,3,12)$ have a triplet quantum yield smaller than 0.45 , and the highest non-radiative decay rate among all samples. For metalloporphyrins $\mathbf{1 8}$ and 19, a drastic decrease in the intersystem crossing time is also observed, following the same behavior as observed for the fluorescence. Moreover, the values of $\phi_{T}$ for metalloporphyrins $\mathbf{1 8}$ and $\mathbf{1 9}$ are moderate, as the $\mathrm{Zn}(\mathrm{II})$ ion exhibits weak spin-orbital coupling [43]. 
Figure 8 displays the triplet quantum yields for the compounds studied. It is plotted as a function of the radiative quantum yield, from the lower to the higher one. Porphyrins 15, 16 and 17 show the highest intersystem crossing quantum yields of all samples, and 2 and 3 exhibit the highest non-radiative quantum yields, $\left(\phi_{n r}=1-\phi_{f}-\phi_{i s c}\right)$.

$<$ Figure $8>$

As observed in Fig. 8, the porphyrins with the higher radiative decay rates were the 5 and $\mathbf{6}$, highlighting the 5 that has a combination of electron donating (methoxy phenyl) and withdrawing (methoxy carbonyl phenyl) groups symmetrically attached in the ortho and para positions of the porphyrin ring giving the greatest $\phi_{r}$ value. This configuration promotes a reduction of the non-radiative and ISC decay rates and, consequently, contributes to the increase of radiative decay rates. The structure of $\mathbf{6}$ is very similar to $\mathbf{5}$, however, a methoxycarbonylphenyl group is substituted by a methoxyphenyl group at the para position. This modification decreases the radiative decay rate. For other molecules we have observed very similar optical properties. For example, the $\mathbf{1}$ and $\mathbf{1 4}$ present practically the same radiative, nonradiative and ISC decay rate. The same behavior is observed for the $\mathbf{1 0}$ and $\mathbf{1 1}$ porphyrins. On the other hand, for $\mathbf{8}$ and 9, a larger difference between their optical properties could be expected due to the presence of an additional cyanophenyl group in 9. However, it appears to possess only moderate electron withdrawing properties [44] and negligible effect on the optical properties.

\section{CONCLUSIONS}

In summary, by monitoring the fluorescence signal induced by a fixed pattern of pulses excitation, the intersystem crossing processes and photophysical characteristics for meso-substituted porphyrins were studied. All compounds investigated were found to exhibit rather small fluorescence quantum yields, particularly the metalloporphyrins. Most of these porphyrins had a triplet quantum yield $<0.7$, due to non-radiative relaxation channels which compete with the intersystem crossing process. Based on the results shown here, porphyrins with methoxyphenyl groups are good candidates for applications in photodynamic therapy and light harvesting.

\section{ACKNOWLEDGEMENTS}

This work was supported by grants from Science Foundation Ireland (14/TIDA/2362 and 13/1A/1894) and a European Commission H2020-MSCA-IF-2014 fellowship to MF (655142 - CONSORT). Financial support from FAPESP (Fundação de Amparo à Pesquisa do estado de São Paulo, processo no : 2011/12399-0 and nº: 2009/11810-8), FAPEMIG (Fundação de Amparo à Pesquisa do estado de Minas Gerais), CNPq (Conselho Nacional de Desenvolvimento Científico e Tecnológico), CAPES (Coordenação de Aperfeiçoamento de Pessoal de Nível Superior) and the Air Force Office of Scientific Research (FA9550-12-1-0028) are acknowledged.

\section{REFERENCES}

1. Bonnett R. Chem. Soc. Rev. 1995; 24: 19-33.

2. Li J, Ambroise A, Sung IY, Diers JR, Seth J, Wack CR, Bocian DF, Holten D, Lindsey JS. J. Am. Chem. Soc. 1999; 121: 8927-8940.

3. Filatov MA, Baluschev S, Ilieva IZ, Enkelmann V, Miteva T, Landfester K, Aleshchenkov SE, Cheprakov AV. J. Org. Chem. 2012; 77: 11119-11131.

4. Drobizhev M, Stepanenko Y, Dzenis Y, Karotki A, Rebane A, Taylor PN, Anderson HL. J. Am. Chem. Soc. 2004; 126: $15352-15353$. 
5. Kim KS, Lim JM, Osuka A, Kim D. J. Photochem. Photobiol. C: Chem. 2008; 9: 13-28.

6. Baskin JS, Yu HZ, Zewail AH. J. Phys. Chem. A 2002; 106: 9837-9844.

7. Lovell JF, Jin CS, Huynh E, Jin H, Kim C, Rubinstein JL, Chan WCW, Cao W, Wang LV, Zheng G. Nature Mater. 2011; 10: 324-332.

8. Senge MO, Fazekas M, Notaras EGA, Blau WJ, Zawadzka M, Locos OB, Ni Mhuircheartaigh EM. Adv. Mater. 2007; 19: 2737-2774.

9. Spangler CW. J. Mater. Chem. 1999; 9: 2013-2020.

10. Zawadzka M, Wang J, Blau WJ, Senge MO. J. Phys. Chem. A 2013; 117: 15-26.

11. Zawadzka M, Wang J, Blau WJ, Senge MO, Photochem. Photobiol. Sci. 2013; 12: 996-1007.

12. Zawadzka M, Wang J, Blau WJ, Senge MO, Photochem. Photobiol. Sci. 2013; 12: 1811-1823.

13. Shirk JS. Opt. Photon. News, 2000; 11: 19-23.

14. Hagan DJ, Van Stryland EW, Soileau MJ, Wu YY, Guha S. Opt Lett. 1988; 13: 315-317.

15. Liu MO, Tai CH, Hu AT, Wei TH. J. Organomet. Chem. 2004; 689: 2138-2143.

16. Ochsner M. J. Photochem. Photobiol. B 1997; 3: 1-18.

17. Ryan, AA, Senge MO. Photochem. Photobiol. Sci. 2015; 14: 638-660.

18. Wohnhaas C, Mailänder V, Dröge M, Filatov MA, Busko D, Avlasevich Y, Baluschev S, Miteva T, Landfester K, Turshatov A. Macromol. Biosci. 2013; 13: 1422-1430.

19. Lakowicz, JR. Principles of Fluorescence Spectroscopy (3rd ed.), Springer: New York, 2006.

20. Gonçalves PJ, Corrêa DS, Franzen PL, De Boni L, Almeida LM, Mendonça CR, Borissevitch IE, Zílio SC. Spectrochim. Acta A Mol. Biomol. Spectrosc. 2013; 112: 309-317.

21. Gonçalves PJ, De Boni L, Barbosa Neto NM, Rodrigues Jr. JJ, Zílio SC, Borissevitch IE. Chem. Phys. Lett. 2005; 407: $236-241$.

22. Sampaio RN, Gomes WR, Araujo DMS, Machado AEH, Silva RA, Marletta A, Borissevitch IE, Ito AS, Dinelli LR, Batista AA, Zílio SC, Gonçalves PJ, Barbosa Neto NM. J. Phys. Chem. A 2012; 116: 18-26.

23. De Boni L, Monteiro CJP, Mendonça CR, Zílio SC, Gonçalves PJ. Chem. Phys. Lett. 2015; 633: 146-151.

24. De Boni L, Franzen PL, Gonçalves PJ, Borissevitch IE, Misoguti L, Mendonça CR, Zilio SC. Opt. Express, 2011; 19: $10813-10823$.

25. Senge MO, Fazekas M, Pintea M, Zawadzka M, Blau WJ. Eur. J. Org. Chem. 2011; 5797-5816.

26. Wiehe A, Shaker YM, Brandt JC, Mebs S, Senge MO. Tetrahedron 2005; 61: 5535-5564.

27. Zawadzka M, Wang J, Blau WJ, Senge MO. Chem. Phys. Lett. 2009; 477: 330-335.

28. Gravier J, Schneider R, Frochot C, Bastogne T, Schmitt F, Didelon J, Guillemin F, Barberi-Heyob M. J. Med. Chem. 2008: 51; 3867-3877.

29. Senge MO, Shaker YM, Pintea M, Ryppa C, Hatscher SS, Ryan A, Sergeeva Y. Eur. J. Org. Chem. 2010; 237258.

30. Notaras EGA, Fazekas M, Doyle JJ, Blau WJ, Senge MO. Chem. Comm. 2007; 2166-2168.

31. Wiehe A, Simonenko EJ, Senge MO, Roeder B. J. Porphyrins Phthalocyanines 2001; 5: 758-761.

32. Senge MO, Ryppa C, Fazekas M, Zawadzka M, Dahms K. Chem. Eur. J. 2011; 17: 13562-13573.

33. Senge MO, Feng X. Tetrahedron Lett. 1999; 40: 4165-4168.

34. Ryan A, Gehrold A, Perusitti R, Pintea M, Fazekas F, Locos OB, Blaikie F, Senge MO. Eur. J. Org. Chem. 2011; 5817-5844.

35. DeRosa MC, Crutchley RJ. Coord. Chem. Rev. 2002; 233-234: 351-371.

36. Brouwer AM. Pure Appl. Chem. 2011; 83: 2213-2228. 
37. De Boni L, Toro C, Hernandez FE. J. Fluoresc. 2010; 20: 197-202.

38. Ha JH, Yoo SI, Jung GY, Paeng IR, Kim YR. J. Mol. Struct. 2002; 606: 189-195.

39. Schuster DI, Cheng P, Jarowski PD, Guldi DM, Luo C, Echegoyen L, Pyo S, Holzwarth AR, Braslavsky SE, Williams RM, Klihm G. J. Am. Chem. Soc. 2004; 126: 7257-7270.

40. Borisevich, EA, ,Solov'ev KN. Phys. Usp. 2005; 48: 231-253.

41. Fonda HN, Gilbert JV, Cormier RA, Sprague JR, Kamioka K, Connolly JS. J. Phys. Chem. 1993; 97: $7024-7033$.

42. Quimby DJ, Longo FR. J. Am. Chem. Soc. 1975; 97: 5111-5117.

43. Harriman A. J. Chem. Soc., Faraday Trans. 1, 1980; 76: 1978-1985.

44. Vivas MG, Silva DL, Malinge J, Boujtita M, Zalesny R, Bartkowiak W, Agren H, Canuto S, De Boni L, Ishow H, Mendonça CR. Scientific Rep. 2014; 4: 4447; doi:10.1038/srep04447. 
Table 1. Photophysical parameters of the studied porphyrin compounds. Fluorescence lifetime $\left(\tau_{f}\right)$, fluorescence quantum yield $\left(\phi_{f}\right)$, radiative $\left(\tau_{r}^{\mathcal{S}}\right)$, non-radiative lifetime $\left(\tau_{n r}^{\mathcal{S}}\right)$, and non-radiative $\left(\phi_{n r}\right)$ quantum yield, intersystem crossing time $\left(\tau_{i s c}\right)$ and triplet quantum yield $\left(\phi_{T}\right)$. Numbers in parentheses give esd's.

\begin{tabular}{|c|c|c|c|c|c|c|c|}
\hline Compound & $\tau_{f}(\mathrm{~ns})$ & $\phi_{f}=\phi_{r}$ & $\tau_{r^{\prime}}^{s}(\mathrm{~ns})$ & $\tau_{n r^{\prime}}^{S}(\mathrm{~ns})$ & $\phi_{n r}$ & $\tau_{\text {isc }}(\mathrm{ns})$ & $\phi_{T}$ \\
\hline 1 & $13.1(5)$ & $0.037(4)$ & $350(40)$ & $39(4)$ & $0.34(4)$ & $21.0(3)$ & $0.60(1)$ \\
\hline 2 & $4.6(2)$ & $0.010(2)$ & $340(50)$ & $7(1)$ & $0.69(1)$ & $14.8(3)$ & $0.30(7)$ \\
\hline 3 & $7.7(2)$ & $0.026(2)$ & $300(50)$ & $13(2)$ & $0.6(2)$ & $20.6(2)$ & $0.37(8)$ \\
\hline 4 & $12.6(2)$ & $0.054(3)$ & $240(20)$ & $30(3)$ & $0.42(4)$ & 24.1(3) & $0.52(7)$ \\
\hline 5 & $11.5(6)$ & $0.074(3)$ & $160(10)$ & $28(1)$ & $0.42(2)$ & $22.7(2)$ & $0.51(3)$ \\
\hline 6 & $12.4(7)$ & $0.058(5)$ & $220(20)$ & $29(2)$ & $0.43(4)$ & 24.1(3) & $0.52(6)$ \\
\hline 7 & $11.5(6)$ & $0.026(2)$ & $440(90)$ & $26(5)$ & $0.44(9)$ & $21.5(4)$ & $0.5(1)$ \\
\hline 8 & $13.4(5)$ & $0.055(2)$ & $250(10)$ & $37(2)$ & $0.36(2)$ & $22.8(3)$ & $0.59(3)$ \\
\hline 9 & $12.5(6)$ & $0.054(4)$ & $230(30)$ & $38(4)$ & $0.33(4)$ & 20.3(4) & $0.6(1)$ \\
\hline 10 & $11.4(3)$ & $0.036(4)$ & $320(40)$ & $24(3)$ & $0.46(6)$ & $22.8(2)$ & $0.50(8)$ \\
\hline 11 & $12.5(6)$ & $0.040(5)$ & $310(40)$ & $29(4)$ & $0.43(6)$ & $23.7(3)$ & $0.53(9)$ \\
\hline 12 & $10.2(3)$ & $0.031(2)$ & $330(20)$ & 19(1) & $0.54(4)$ & $23.4(3)$ & $0.43(4)$ \\
\hline 13 & $11.4(6)$ & $0.017(2)$ & $670(80)$ & $22(3)$ & $0.52(7)$ & $25.2(3)$ & $0.45(7)$ \\
\hline 14 & $14.8(9)$ & $0.038(2)$ & $380(20)$ & $41(2)$ & $0.36(2)$ & $24.6(2)$ & $0.60(4)$ \\
\hline 15 & $12.0(5)$ & $0.053(6)$ & $230(10)$ & $48(3)$ & $0.25(2)$ & $17.2(4)$ & $0.70(6)$ \\
\hline 16 & $14.4(4)$ & $0.038(3)$ & $380(30)$ & $68(6)$ & $0.21(2)$ & $19.1(4)$ & $0.75(8)$ \\
\hline 17 & $13.4(4)$ & $0.035(2)$ & $384(20)$ & $88(5)$ & $0.152(9)$ & $16.5(2)$ & $0.81(3)$ \\
\hline 18 & $3.1(10)$ & $0.033(3)$ & $95(9)$ & $6.2(7)$ & $0.50(5)$ & $6.7(2)$ & $0.46(6)$ \\
\hline 19 & $2.9(1)$ & $0.025(3)$ & $120(10)$ & $5.4(8)$ & $0.53(7)$ & $6.6(2)$ & $0.44(8)$ \\
\hline
\end{tabular}




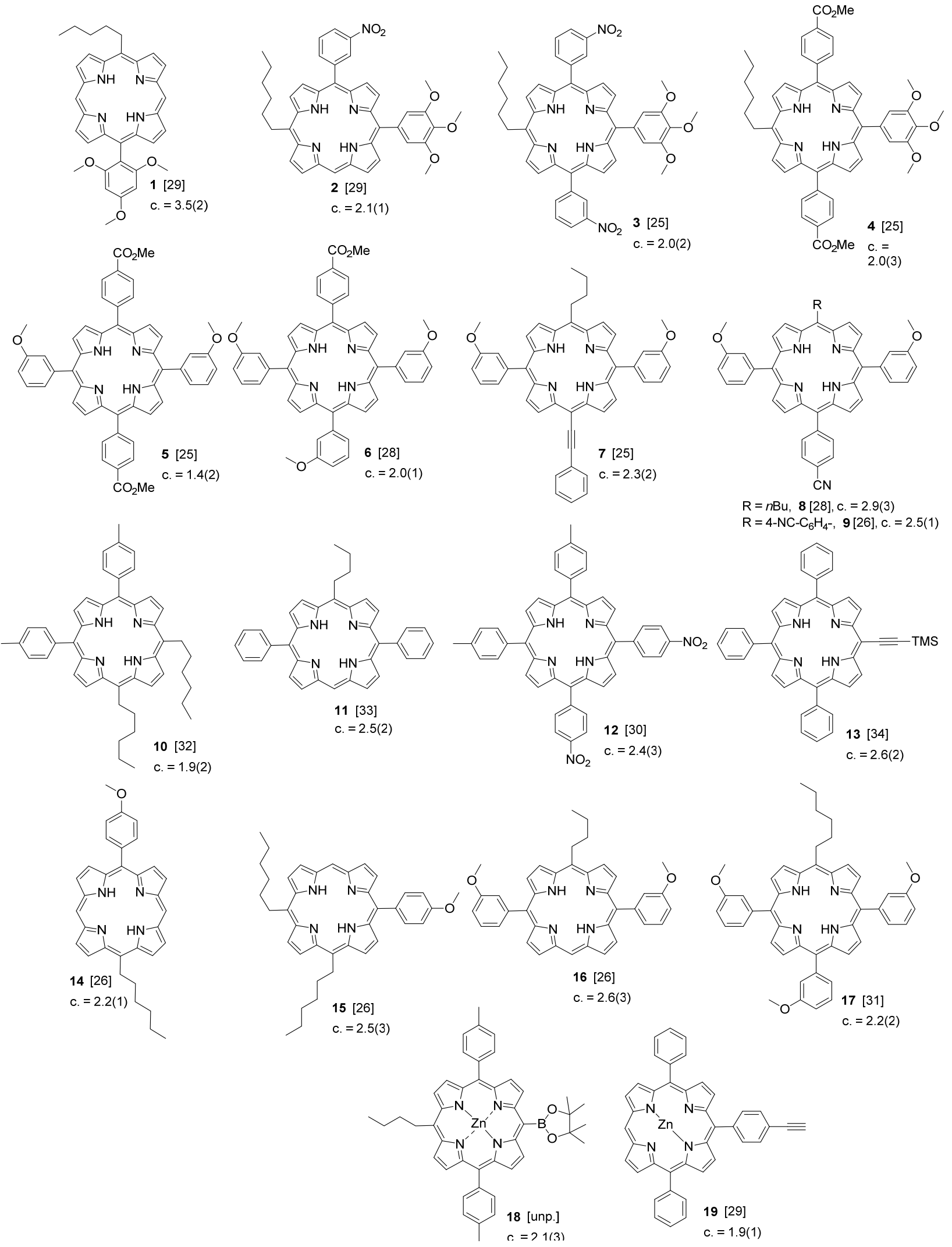

Fig. 1. Molecular structures of porphyrin materials studied. Sample concentrations used to measure optical processes are given in $10^{17}$ molecules $/ \mathrm{cm}^{3}$ (numbers in parentheses give e.s.d.'s). References for synthetic procedures are given in brackets. 

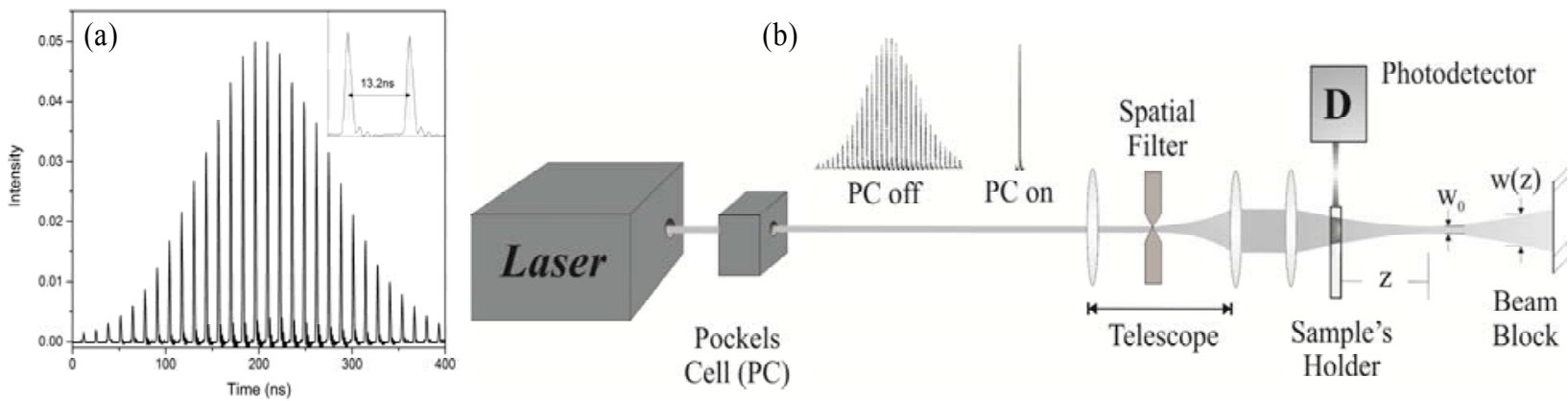

Fig. 2. (a) Pulse train characteristic profile used as excitation source. In the upper right corner the regular temporal distance between two successive pulses is highlighted. (b) Experimental setup. 


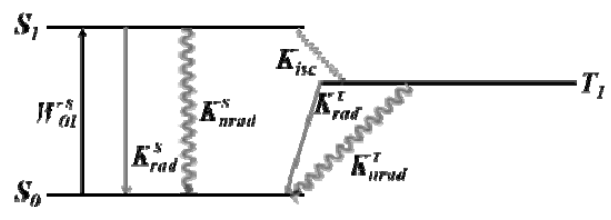

Fig. 3. Jablonski three level diagram, representing ground $\left(S_{0}\right)$, first excited $\left(S_{l}\right)$ and a triplet excited state $\left(T_{l}\right)$. Radiative transitions are depicted by solid arrows, whereas radiation-less transitions are shown as dashed arrows. 
a)
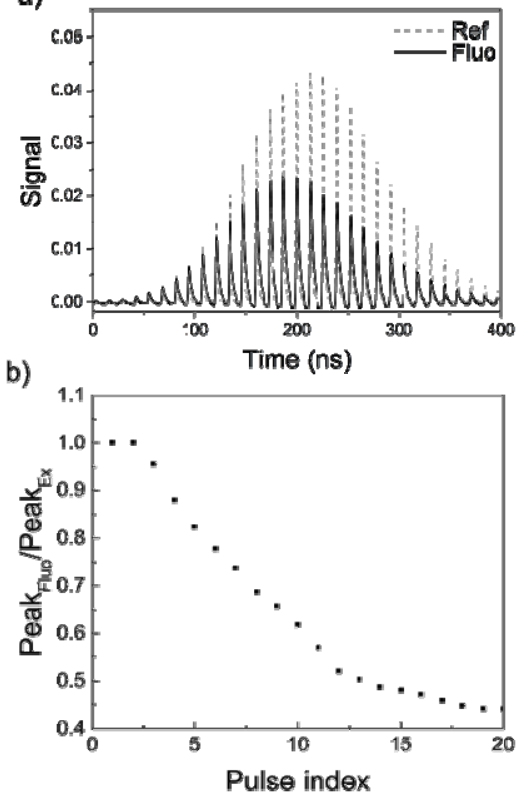

Fig. 4. a) The excitation pulse train reference (grey dashed curve) and the fluorescence induced in the sample by the pulse train envelope (solid black curve); b) Peak $\mathrm{fluo}_{\mathrm{P}} / \mathrm{Peak}_{\mathrm{ex}}$ signal obtained by the ratio between the fluorescence signal with respect to pulse excitation (reference), both presented in a). 


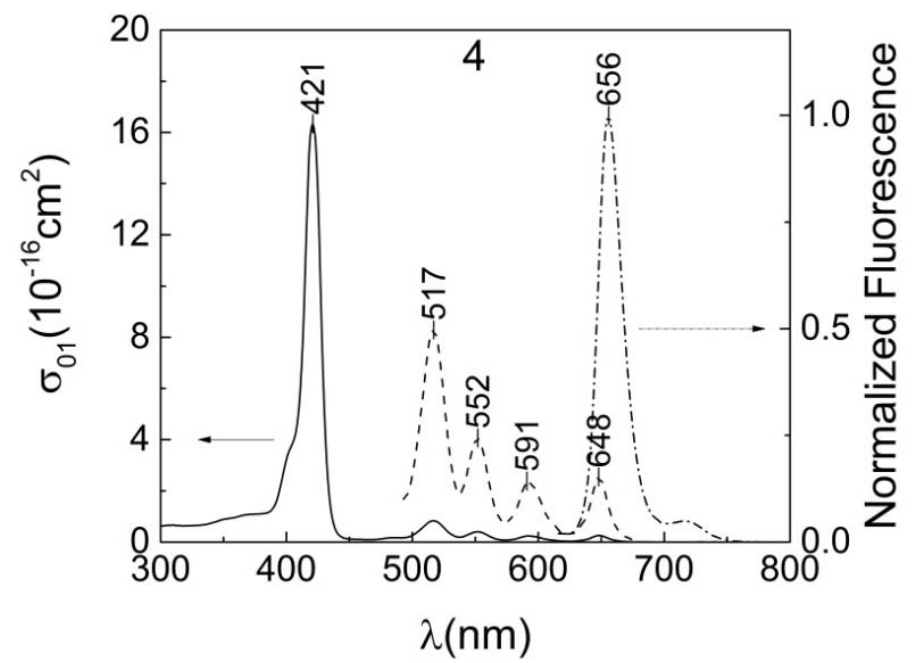

Fig. 5. Absorption cross-section spectrum of the transition (solid black curve - left scale) and normalized fluorescence spectrum (dash-dot curve - right scale). The dashed black curve represents the absorption $(\times 10)$ cross section in the Q-band region. 


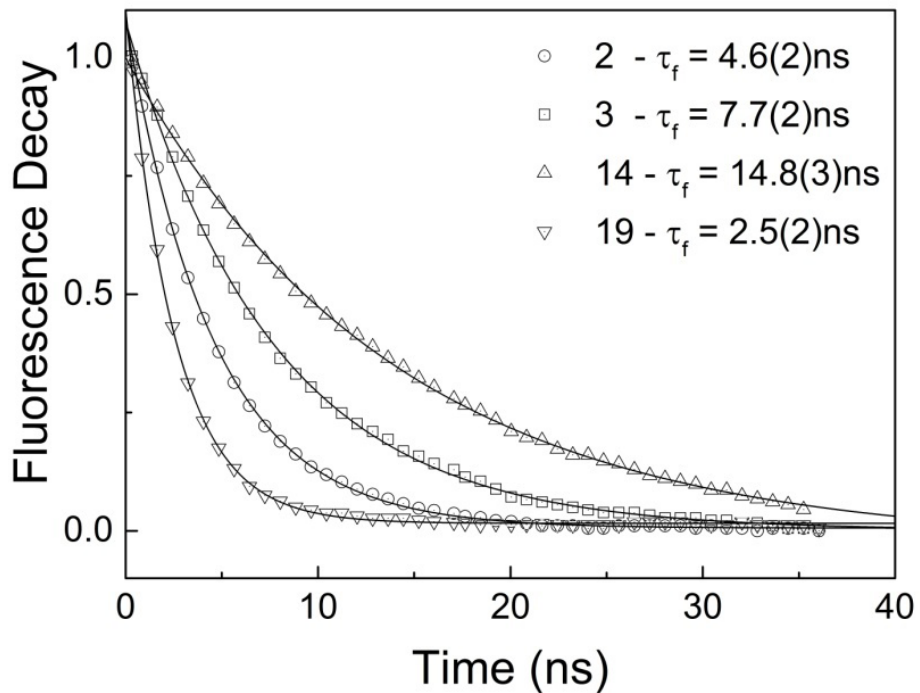

Fig. 6. Fluorescence decay curves (symbols) for four samples $(2,3,14$ and 19). Each curve was fitted by a single exponential decay function. Numbers in parentheses gives esd's. 


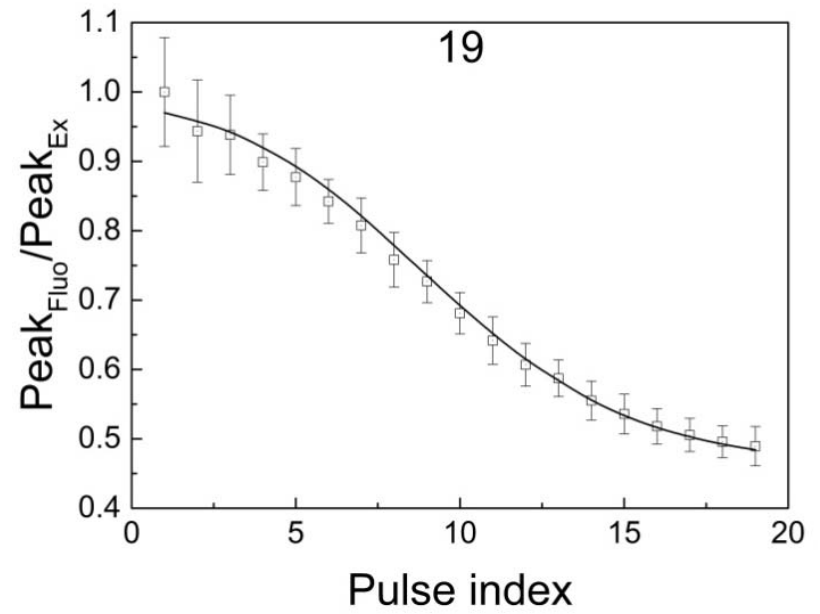

Fig. 7. The peak to peak ratio of fluorescence and excitation representing the depletion of fluorescence as a function of the pulse index within the pulse train. 


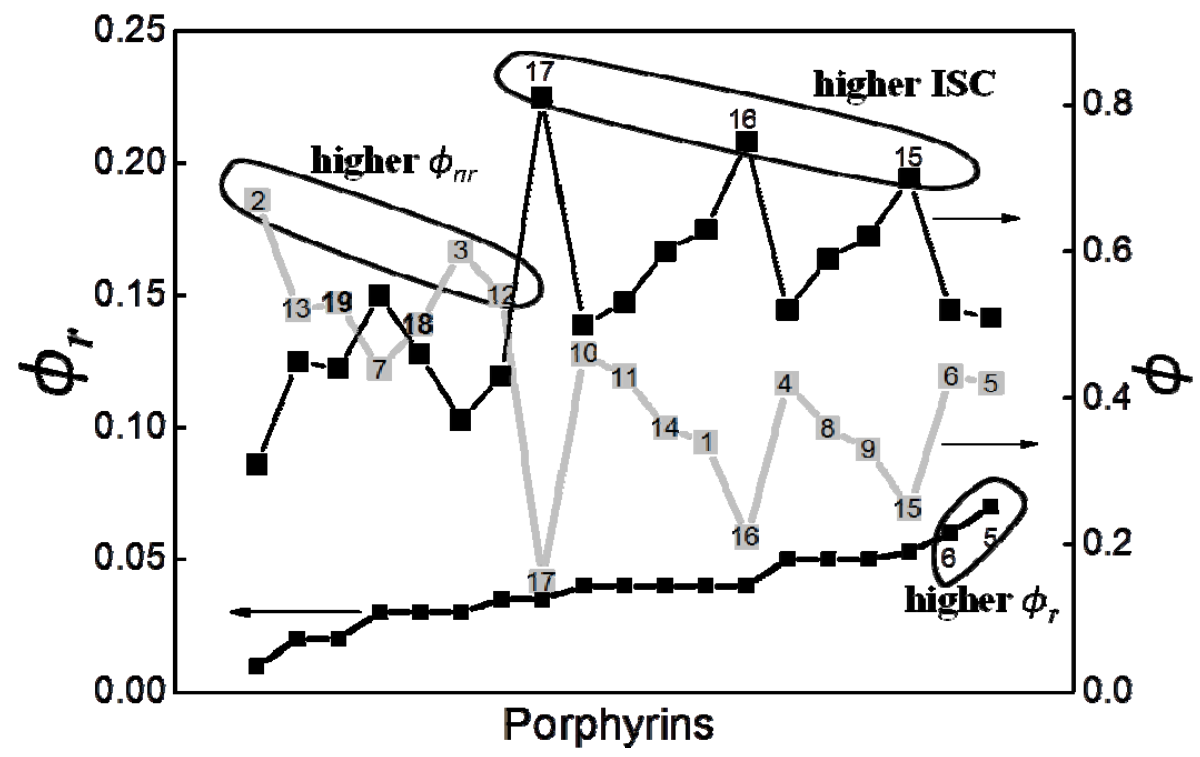

Fig. 8. Radiative (left axis) and non-radiative decay (right axis) rates and triplet quantum yield (right axis) for each porphyrin. The parameters were plotted ordering the porphyrins on the $\mathrm{x}$-axis according to increasing radiative quantum yield. 